\title{
NUMERICAL ANALYSIS OF OSCILLATORY FLOWS IN THE HUMAN BRAIN BY A LATTICE-BOLTZMANN METHOD
}

\author{
SEONG-RYONG KOH ${ }^{1}$, JONG-HYUN KIM ${ }^{2}$ AND ANDREAS LINTERMANN ${ }^{3}$ \\ ${ }^{1}$ Jülich Supercomputing Centre \\ Forschungszentrum Jülich, Wilhelm-Johnen-Straße, 52425 Jülich, Germany \\ Email: s.kohefz-juelich.de \\ https://www.fz-juelich.de/ias/jsc \\ ${ }^{2}$ Korea University Medicine \\ Korea University Guro Hospital, 148 Gurodong-ro Guro-gu, 08308 Seoul, Korea \\ http://guro.kumc.or.kr/language/ENG/main/index.do \\ ${ }^{3}$ Jülich Supercomputing Centre \\ Forschungszentrum Jülich, Wilhelm-Johnen-Straße, 52425 Jülich, Germany \\ https://www.fz-juelich.de/ias/jsc
}

Key words: cerebrospinal fluid, brain ventricular system, lattice-Boltzmann method

\begin{abstract}
The cerebrospinal fluid flow in a brain ventricular system is analyzed by the numerical approach employing a lattice-Boltzmann (LB) method. The cerebrospinal fluid, which surrounds the human brain and spinal cord, fills the cerebral ventricles as well as the cranial and subarachnoid spaces. Diseases in a central nerve system disrupt the flow circulation which influences on a number of vital functions. A computational fluid dynamics technique is used to determine the member geometry impact on the flow motion. The numerical analysis focuses on building a simulation-based basis for testing/optimizing therapeutical methods and understanding the pathophysiology. Magnetic resonance (MR) imaging is exploited to obtain realistic geometries in a brain ventricular system. The computational domain is discretized by a hierarchical Cartesian octree mesh. The numerical procedure based on an LB method overcomes the difficulties raised by typical finite-difference and finite-volume methods on high-performance computing (HPC) systems. An oscillating flow boundary condition is defined to resolve the kinetic behavior of cerebrospinal fluid in a cardiac cycle. The three-dimensional structures captured in the cerebral ventricles show a qualitative agreement with an observation based on an MR velocity mapping. The simulation on a HPC system is able to provide further insights into the transport from brain to spinal cord.
\end{abstract}

\section{INTRODUCTION}

The cerebrospinal fluid (CSF) fills the cerebral ventricles as well as the cranial and subarachnoid spaces (SAS). Circulating in the brain and the spinal cord, the CSF transports nutrients and neuroendocrine substances and removes toxic chemicals to preserve the healthy environment inside a brain ${ }^{1}$. The flow pulsates through two lateral ventricles, which are connected with the third ventricle. A thin channel, i.e., the aqueduct of Sylvius, communicates with the third and the fourth ventricle. The CSF is transferred 
from the fourth ventricle to the cranial and spinal SAS. Diseases in the central nerve system immediately impacts on a number of vital functions, e.g., disruption in CSF circulation. In clinic the intracranial pressure (ICP) is monitored in neurosurgical practice since the ICP variation in time reflects pathological symptoms associated with CSF pressure changes. The values are used in diagnosis and the treatment of diseases inside the cerebral flow network ${ }^{2,3}$. The baseline pressure level is affected by periodic components coming from the cardio-respiratory activity. Fluctuation of mean arterial pressure with heart rate causes small amplitude rapid pulsation, and respiration causes larger amplitude fluctuations of lower frequency. ICP is completely described only by information about both the baseline level and the pulsating components ${ }^{2}$. Several prominent disease states deduce from disorders of the CSF dynamics. This includes, e.g., an increased intracranial pressure by hydrocephalus ${ }^{4}$ or decreases in the flow and pulsatility by Chiari malformations ${ }^{5}$. However, the clinical decision making is difficult when the symptom occurs with no disease characteristics as observed in normal pressure hydrocephalus. Unfortunately, the specific etiologies and the biomechanical interactions between the components of the central nerve system (CNS) are hardly understood under abnormal fluid conditions

The basic physiologic concept about the CSF and ICP started already in the early 19th century. So called the Monro-Kellie doctrine ${ }^{6,7}$ is formalized as the fundamental concept reads

$$
V_{\mathrm{CSF}}+V_{\text {Blood }}+V_{\text {Brain }}+V_{\text {Other }}=V_{\text {Intracranial space }}=\text { constant }
$$

where $V_{\mathrm{CSF}}$ is volume of CSF, $V_{\mathrm{Blood}}$ is volume of blood, $V_{\mathrm{Brain}}$ is volume of brain, and $V_{\mathrm{Other}}$ is volume of any abnormal component, such as a tumor. This equation provides a general framework for under-

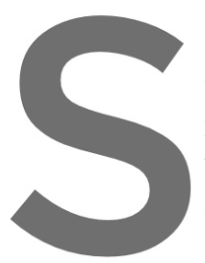
standing pathologic cauls of cerebrospinal fluid is is water. The water soluti blood cells. The major port within sections of the except for the cerebral aqueduct and the frontal
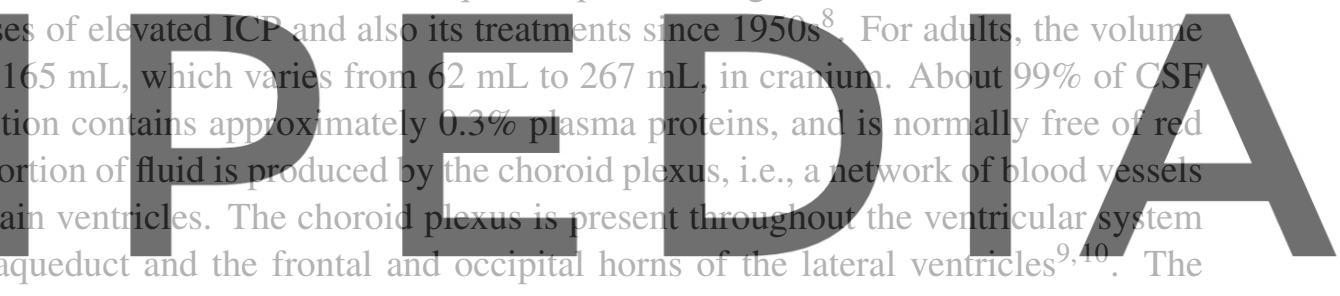

CSF production starts from a filtered form of plasma with movement guided by a difference in pressure

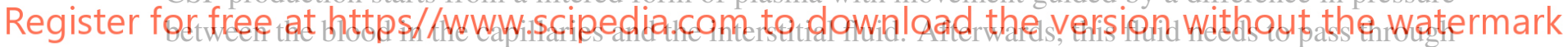

the epithelium cells lining the choroid plexus into the ventricles. An active process requires the transport

of sodium, potassium and chioride that draws water into CSF by creating osmotic pressure. And this transcellular fluid is constantly absorbed again such that only a certain amount of fluids is present at any one time. An equivalent circuit model of the flow network described the formation, storage, and the absorption at a given intracranial pressure ${ }^{11}$. According to this model the intracranial pressure value can be determined by the CSF formation current, a single resistance element, and the pressure in the dural sinuses. As introduced in the previous slides, the ICP signal has a baseline value and the amplitude of the fluctuations remain almost constant at a normal condition.

The Monro-Kellie hypothesis implies that a brain disease due to abnormal components makes reciprocal changes in the volumes of brain, blood, and cerebrospinal fluids to preserve the ICP. The main motivation of the current study is to determine the CSF motion based on the fundamental hypothesis. Furthermore, in surgical practice the external or the internal drainage is chosen as treatment to control the high ICP values $^{12}$. Although doctors also use other methods with medication, the treatment of hypertension is typically mechanical for the fluid volume. The detailed fluid dynamic analysis is able to provide a better understanding of the CSF flow behavior in the complex brain ventricular system. Computational fluid 
dynamics can be used to quantify the functional interactions of the members of the ventricular system.

In the aforementioned clinical applications $\mathrm{s}^{8,11,12}$ the fluid circulation models consider the pressure dynamics and the dynamics of CSF and cerebral blood flow. The quantitative assessment provides robust evidences in judging a patient care when the clinical parameters are subtle and ambiguous ${ }^{1,13}$. The fluid dynamics model exploited in the numerical analyses is based on the fundamental conservation law of mass, momentum, and chemical reactions ${ }^{14-16}$. The approaches using computational fluid dynamics (CFD) can simulate realistic CSF flows to predict the patient-specific scenarios. The present study aims to establish a basis to test and optimize a clinical therapy via the anatomically accurate simulation based on a robust numerical procedure.

The three-dimensional images obtained by magnetic resonance imaging (MRI) provide the structures of ventricles and the SAS. For a normal healthy human the CSF speed ranges in several centimeters per second. The typical Reynolds number is in the order of $O\left(10^{2}\right)$. However, the physiological mechanisms of the CSF circulation develop vortical flow structures which exhibit a turbulent-like motion induced by the difference in systolic and diastolic velocities and the interactions of micro-structures with fluids ${ }^{15,16}$. To resolve the micro-structures, like nerve roots and trabeculae, a short spinal segment already demands a significant increase of the computational cost ${ }^{15}$. These fluid dynamic and the computational difficulties obstruct the development of a therapeutic basis in medical diagnosis.

In the present numerical study, the complex anatomical geometries as well as the CSF pulsatility are tackled with the high-resolution computations using the high-performance computing (HPC) technique. The numerical solutions of transitional phases will be validated on the quantitative assessment based on literature ${ }^{13,17}$. The impact of pulsatility on the flow
configuration such that the fundamental findings
outcomes of this study have the potential to be expa
numerical models of CNS. Together with the clini
enable a better understanding of the pathophysiolog
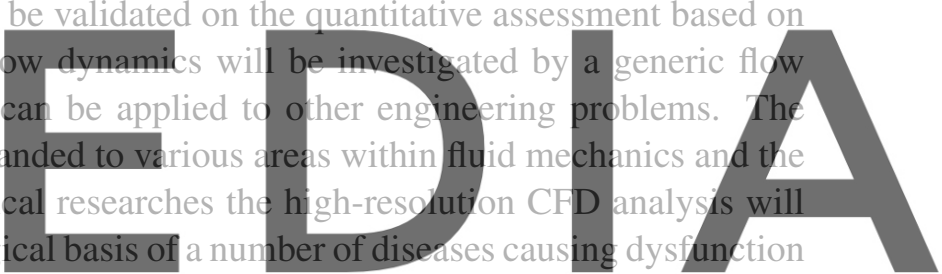

of CSF circulation.

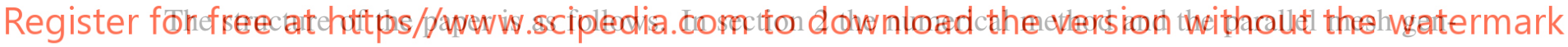
eration are described. Then, the geometries obtained by MR image manipulation in section 3 and the flow configuration in section 4 are defined. Subsequently, the numerical result of the high resolution numerical method is discussed in section 5. Finally, the major findings are summarized in section 6.

\section{NUMERICAL METHODS}

\subsection{Lattice-Boltzmann method}

The simulation of CSF flows is performed with a lattice-Boltzmann (LB) method in the quasiincompressible flow regimes. By solving the microscopic distribution of the fluid particles the LB method can determine flow fields in a simpler manner than the typical finite-difference and finite-volume approaches. The LB algorithm is completely local operation and its locality ensures a massive parallelism on the high-performance computing (HPC) system. The LB method is validated in generic flow configurations $^{18,19}$ and has been applied to bio-fluid mechanical problems ${ }^{20,21}$.

In the single-relaxation-time (SRT) LB method, the Boltzmann equation with the Bhatnagar-GrossKrook (BGK) approximation of the right-hand-side collision term ${ }^{22}$ is solved for the particle probability 
distribution functions $f_{i}$ (PPDFs) in a discretized form

$$
f_{i}\left(\mathbf{x}+\xi_{i} \Delta t, t+\Delta t\right)=f_{i}(\mathbf{x}, t)-\omega_{S R T}\left(f_{i}(\mathbf{x}, t)-f_{i}^{e q}(\mathbf{x}, t)\right),
$$

where $\mathbf{x}=\left(x_{1}, x_{2}, x_{3}\right)^{T}$ and $\xi_{i}=\left(\xi_{1}, \xi_{2}, \xi_{3}\right)^{T}$ are the position and molecular velocity vectors, $t$ is the time and $\Delta t$ its increment. The discrete Maxwellian distribution function $f_{i}^{e q}$ is determined by

$$
f_{i}^{e q}(\mathbf{x}, t)=\rho t_{p}\left[1+\frac{u_{a} \xi_{i a}}{c_{s}^{2}}+\frac{u_{a} u_{b}}{2 c_{s}^{2}} \cdot\left(\frac{\xi_{i a} \xi_{i b}}{c_{s}^{2}}-\delta_{a b}\right)\right]
$$

This discretized formulation consists of a fluid density $\rho$, direction-dependent weighting factors $t_{p}$, an isothermal speed of sound $c_{s}=1 / \sqrt{3}$, velocity components $u_{a}$ and $u_{b}$ of the fluid velocity vector $\mathbf{u}$, and the Kronecker delta $\delta_{a b}$ with $a, b \in\{1,2,3\}$. The quantity $\omega_{S R T}$ is the relaxation factor

$$
\omega_{S R T}=\frac{c_{s}^{2}}{v+\Delta t c_{S}^{2} / 2}
$$

with viscosity v. The parameter $i$ in Eqs. (2) and (3) is chosen by the D3Q27 discretization scheme ${ }^{23}$ with 27 direction in a three dimensional flow field. The macroscopic variables can be obtained from the moments of the PPDFs ${ }^{24}$
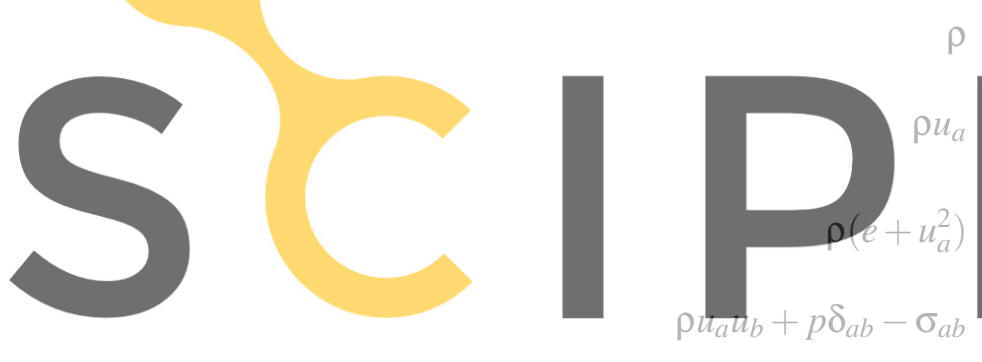

$$
\rho=\sum f_{i}(\mathbf{x}, t)
$$
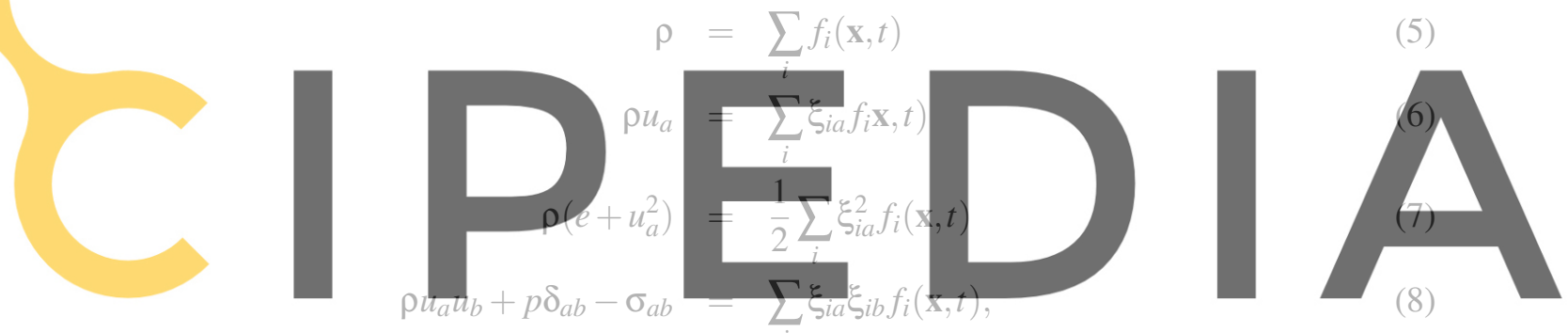

Register for free at hthtps//nwwyscripedia.com

$$
p=\rho c_{s}^{2}
$$

The computations are performed in non-dimensional space. The non-dimensional molecular velocity components $\xi_{i a}=\tilde{\xi}_{i a} / \tilde{\xi}_{0}$ are obtained from the dimensional molecular velocity components $\tilde{\xi}_{i a}$ and the reference molecular velocity $\tilde{\xi}_{0}$. The non-dimensionalization of the density $\rho=\tilde{\rho} / \tilde{\rho}_{0}$ uses the reference density $\tilde{\rho}_{0}$. The reference fluid velocity $U_{0}$ in the simulation is obtained from the Mach number $\mathrm{Ma}=$ $U_{0} / c_{s}$ used in the LB simulation. The viscosity in the simulation is obtained by means of the Reynolds similarity, i.e, the Reynolds number $\operatorname{Re}=U_{0} \tilde{L} / \tilde{\mathrm{v}}$ with reference velocity magnitude $U_{0}$, reference length $\tilde{L}$ and fluid viscosity $\tilde{v}$ are used to obtain the non-dimensional viscosity via

$$
\mathrm{v}=\tilde{\mathrm{v}} \cdot \mathrm{Ma} \cdot \frac{c_{s}}{U_{0}} \cdot \frac{L}{\tilde{L}}
$$

where $L=\tilde{L} / \Delta \tilde{x}$. The relation of the pressure uses Eq. (9) for conversion. All results presented in Sec. 5 are in their non-dimensional form. 


\subsection{Grid generation}

A hierarchical Cartesian computational mesh is generated using the massively parallel method presented $\mathrm{in}^{25}$. That is, each message passing interface (MPI) process initially generates a cube, in the following referred to as cell, around the geometry with an edge length of the maximum extent of the geometry in the three Cartesian coordinate axes. Subsequently, this cube is subdivided into eight child cells constituting an octree with parent-child relationships between the initial cell on level $l_{0}$ and its descendants on level $l_{1}$. Cells outside the geometry are identified and removed from the tree. The subdivision process then recursively continues the refinement up to a user-defined level $l_{m}$. On $l_{m}$, all levels $l_{j}(j<m)$ are removed and a Hilbert curve is placed in the remaining cells. The computational mesh is then equally distributed among the MPI processes by cutting the Hilbert curve with respect to the Hilbert identifier. Thereby, each process keeps only those cells that have been assigned to it.

In a parallel algorithm each MPI process subsequently starts to continue the refinement up to a userdefined level $l_{n}$. Finally, neighborhood information across MPI ranks is globally restored and a cell ordering is introduced following the Hilbert curve on level $l_{m}$ and a $z$-ordering, i.e., a depth-first ordering, for all levels $l_{k}(m<k \leq n)$. Process imbalances are treated by a load-balancing technique. For further details, the reader is referred to ${ }^{25}$. The geometry of brain ventricles is obtained by manipulation of MRI scans. The procedure of medical images is described in the following section 3.

\section{GEOMETRY}

The flow simulation of a brain ventricular system is performed to establish a basis for supporting the diagnostic procedure. The understanding of pathology and the biofluid dynamics is able to improve
the therapeutic methods. In the flow simulation the geometry of ventricles and subarachnoid space is
obtained by segmentation of cerebrospinal flyid regions. The fluid is identified by the T1-weighted MRI
scans shown in Fig. 1. The MRI scan using the SIMENS Magnetom-Skyra examined the main ranial
nerves region of a nornal adult. The personal data is anonymized. The three-dimensional MRI scan

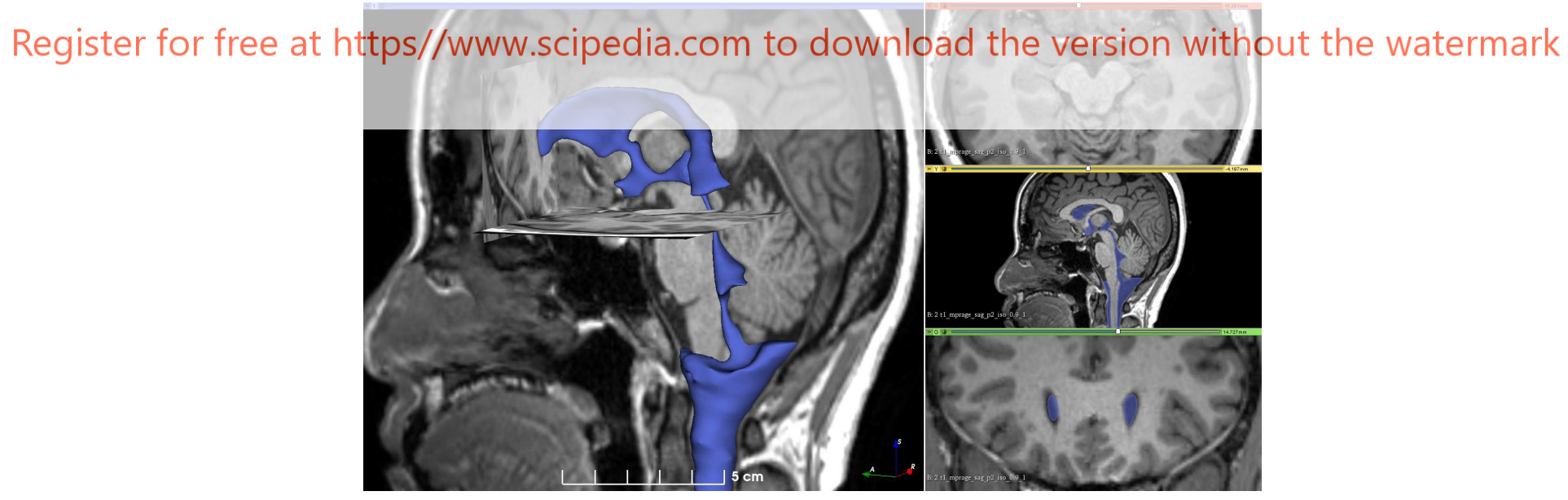

Figure 1: Segmentation of a brain ventricular system visualized by the T1-weighted MRI scans; in the left a main window shows the blue three-dimensional structure used in the flow simulation, and in the right three sub-windows show the segmentation colored by blue. 

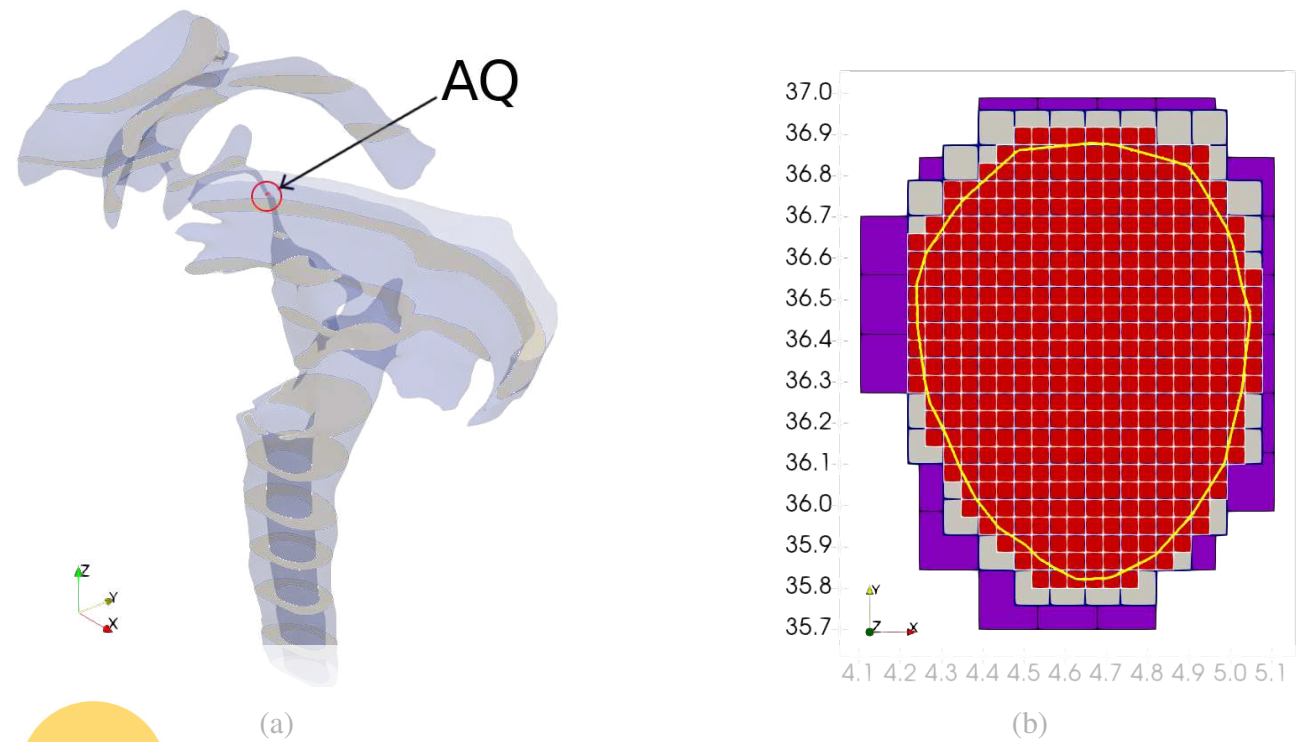

(b)

Figure 2: Geometry of brain ventricles obtained by segmentation of CSF region, (a) brain ventricles and subarachnoid space, (b) an enlarged view of meshes in the cross-section (AQ) at the aqueduct of Sylvius where the unit of length is the millimeter (mm).

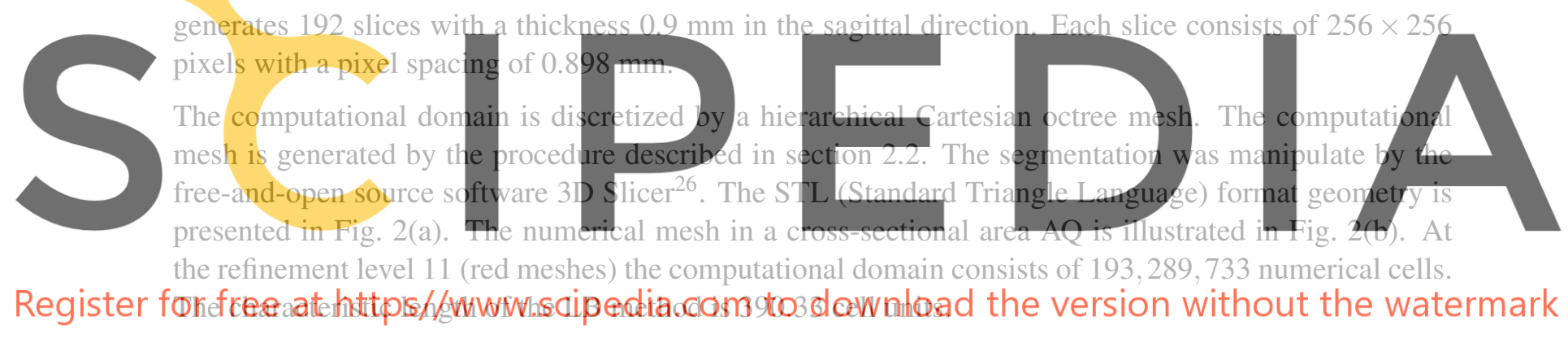

\section{FLOW CONFIGURATION}

The MRI manipulation generates a realistic geometry of a brain ventricular system. The commonly faced issues in studying biofluid dynamics are boundary conditions of a finite computational domain. The variation of intracranial pressure is an essential parameter for the flow configuration. In the following section the configuration of a ventricular system is detailed with model geometries and an oscillating flow boundary condition.

\subsection{Flow oscillation and non-dimensional frequency}

The Monro-Kellie doctrine implies that a brain disease due to abnormal components makes reciprocal changes in the volumes of brain, blood, and cerebrospinal fluids to preserve the intracranial pressure. The flow simulation is configured by the pressure variation as an only factor that impacts on the CSF dynamics. The pressure values at the inflow and the outflow boundaries are determined by the steady flow condition with the constant flow properties. Since the flow motion in brain ventricles is oscillating 


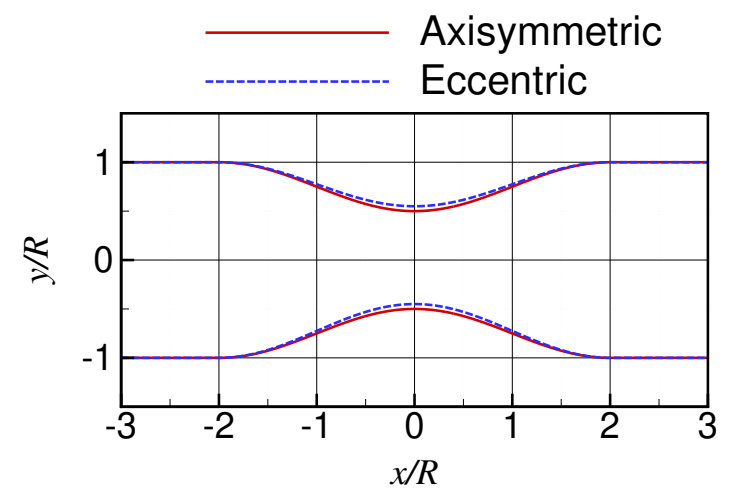

Figure 3: Profiles of an axisymmetric and an eccentric pipe near the stenosis at the $x$-coordinate $x=0$, the profiles are scaled by a radius $R(=D / 2)$.

with respect to the cardiac cycles. The oscillation frequency is defined by Womersley number Wo

$$
\mathrm{Wo}_{\mathrm{o}}=L \sqrt{\frac{\omega \rho}{\mu}},
$$

where $\omega=2 \pi f$ is angular frequency, $\mu$ is the dynamic viscosity, and $\rho$ is the density of fluid. In the present study the pipe diameter $D$ is chosen as a reference length $L$. Concerning a reference velocity $U$ a non-dimensional frequency St can be calculated using Eq. (11) as following
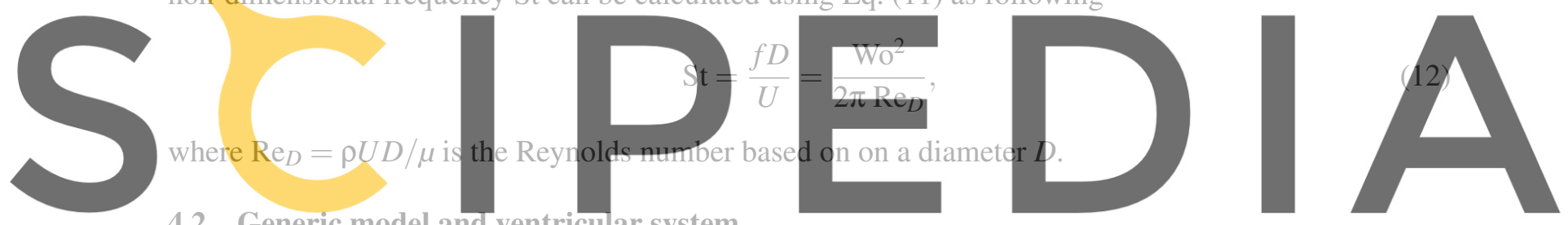

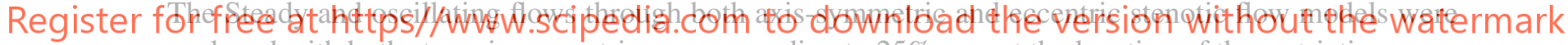
analyzed with both stenosis geometries corresponding to $25 \%$ area at the location of the restriction compared to the inflow area. The baseline stenosis geometry is similar to that used in the stenotic flow experiments ${ }^{27}$. The profiles of the axis-symmetric and eccentric stenosis models are defined by following equations. A cosine function dependent on the axial coordinate $x$ is used to generate the geometry. The cross-section coordinates $y$ and $z$ are determined by using $S(s)$ specifying the shape of the stenosis by

$$
S(x)=R\left[1-s_{0}(1+\cos (2 \pi x / L))\right], \quad E(x)=0.1 s_{0}[1+\cos (2 \pi x / L)],
$$

where $R$ is the radius of the non-stenotic pipe, $s_{0}=0.25$ realizes the $75 \%$ area reduction, and $L=4 R$ is the length of the stenosis. By introducing the eccentricity $E(x)$, the $y$ and $z$ coordinates are defined by

$$
y=S(x) \cos \theta+E(x), \quad z=S(x) \sin \theta,
$$

at the azimuthal angle $\theta$ on the $y z$-plane. The axis-symmetric and the eccentric pipe are defined by Eqs. (13) \& (14) with a 5\% eccentricity at $x / R=0$. In Fig. 3 the cross-section profiles of a axisymmetric and a eccentric pipe is illustrated near the stenotic region at $x=0$. 
For the ventricular system the CSF oscillation in brain ventricles possesses pressure variation in a cardiac cycle. The temporal variation of pressure is defined at the lateral ventricles. The Reynolds number $\operatorname{Re}_{D}$ is defined by a hydraulic diameter ca. $16.7 \mathrm{~mm}$ near the exit of the 4th ventricle. The peak Reynolds number is ca. 628 at the Womersley number Wo $=15^{28}$

\section{RESULTS}

The numerical analysis focuses on the CSF motion which is determined by an oscillating flow boundary condition with the geometry of brain ventricular system connected to the cranial and the spinal SAS. At the beginning two isolated stenotic pipes are considered with the median aperture which mimics drainage from the ventricles to the subarachnoid space.

\subsection{Isolated stenotic pipe flow}

The numerical analysis using a generic configuration is performed to detail the instantaneous and statistical flow features which occur downstream of a stenosis under the oscillating flow condition. The non-dimensional parameters defined in Section 4 are chosen to provide a fundamental basis for understanding the flow dynamics generated by even more complex occlusions within the ventricle system and SAS in the human CNS. The peak Reynolds number is 600 based on the pipe diameter. The flow oscillation is defined by a sinusoidal function at the Womersley number Wo $=15$ scaled by the pipe diameter. In Fig 4 the contours of density and velocity distributions are presented at the peak pressure on the right boundary surface. The axisymmetric configuration develops a symmetric form of shear layers. The flow

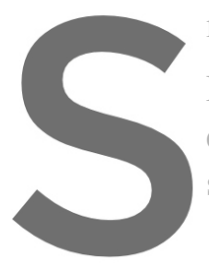
mixing in Fig. 4(b) is pron
Figure 5 shows the impact
eccentric pipe the turbulen
shifted to the wall compar

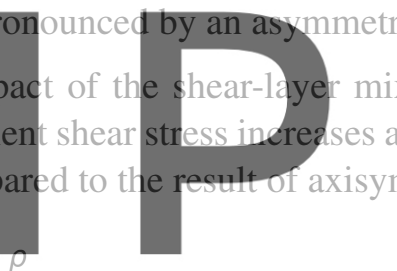

0.998

1.004

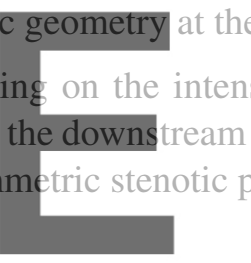

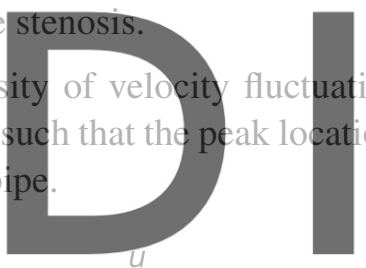

$-0.025 \quad 0.000 \quad 0.025$

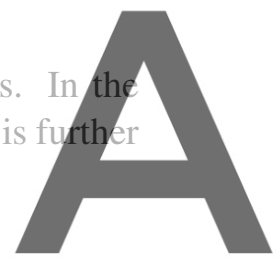

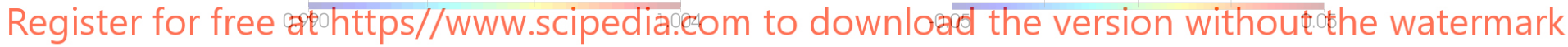
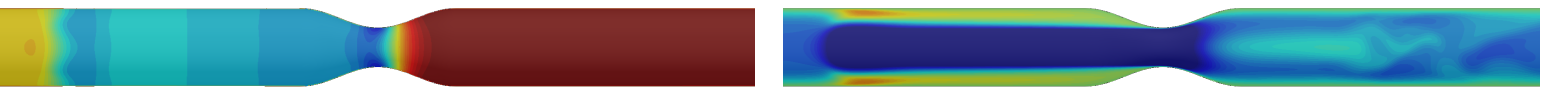

(a) Axisymmetric pipe
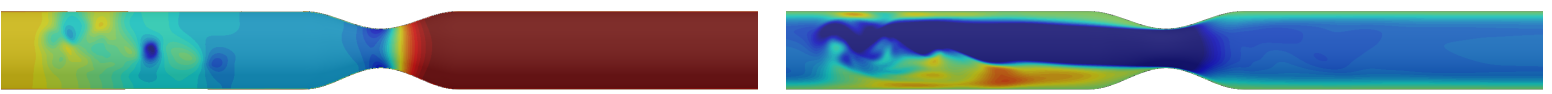

(b) Eccentric pipe

Figure 4: Contours of density and velocity component determined by an axisymmetric and an eccentric stenotic pipe, (a) axisymmetric pipe, (b) eccentric pipe. 

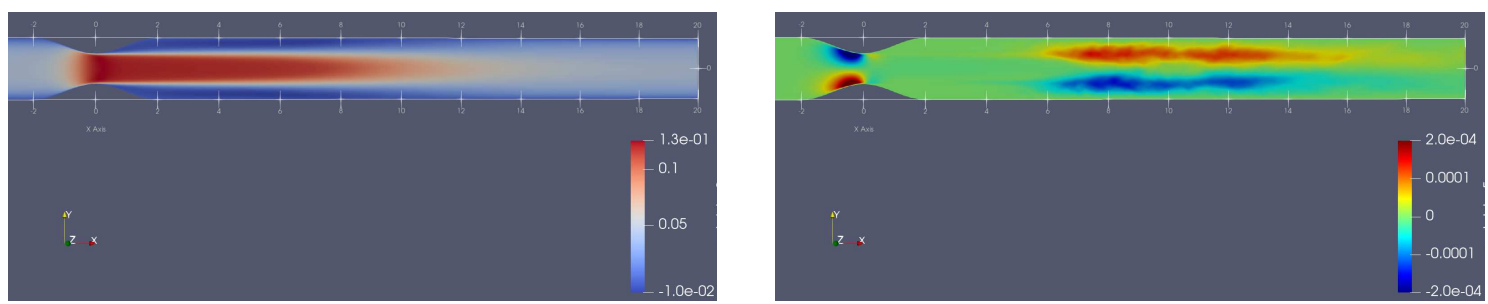

(a) Axisymmetric pipe (left: $\bar{u}$, right: $\overline{u^{\prime} v^{\prime}}$ )
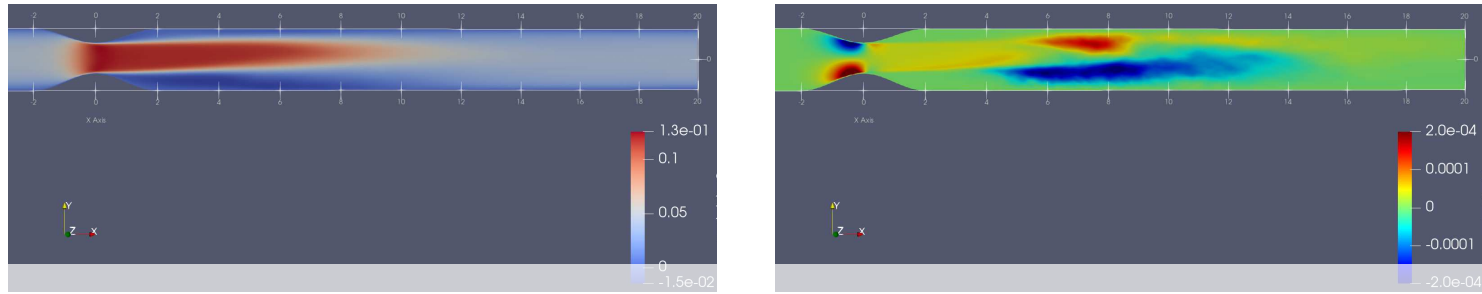

(b) Eccentric pipe (left: $\bar{u}$, right: $\overline{u^{\prime} v^{\prime}}$ )

Figure 5: Contours of time-averaged $u$-velocity and a component of turbulent shear stresses $\overline{u^{\prime} v^{\prime}}$, (a) axisymmetric pipe, (b) eccentric pipe.

\subsection{CSF motion inside ventricles}

In the CSF flow simulation the hydraulic diameter $16.7 \mathrm{~mm}$ is $\phi$ btaind at a cros-section bet $\mathrm{w}$

ventricle and the central ca

diameter at the aqueduct of Sylvius is equivalent to the

The variation of intractanial pressure is correlate

pressure oscillation in the

analysis. In section 5.1 the smat1 eccentricity in

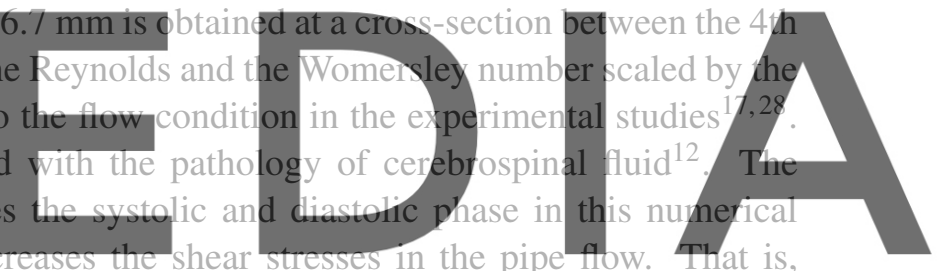

the well-defined geometries of a brain ventricular system are required to predict the CSF flow field in

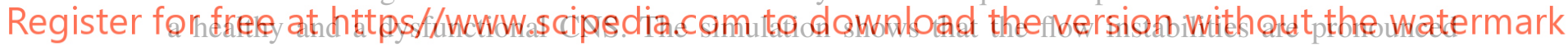

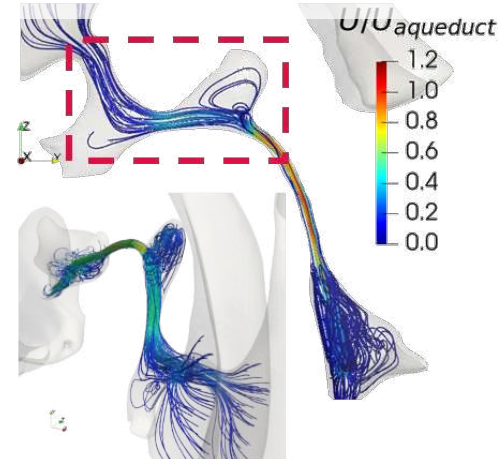

(a) Systole

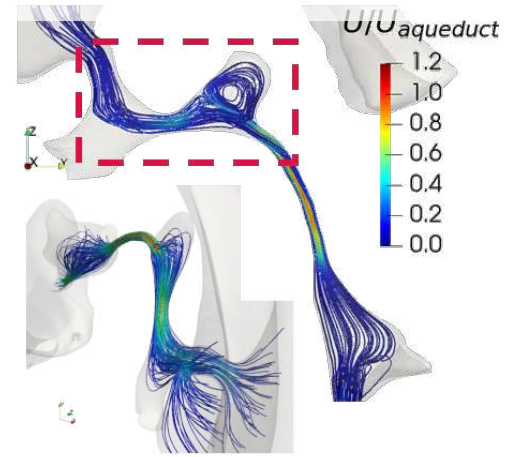

(b) Diastole

Figure 6: Streamlines in the 3rd ventricle, the color indicates a velocity magnitude normalized by the mean velocity of the aqueduct of Sylvius, (a) systole phase, (b) diastole phase. 


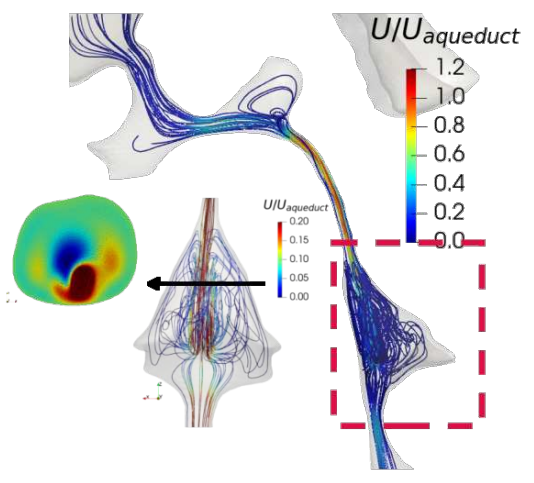

(a) Systole

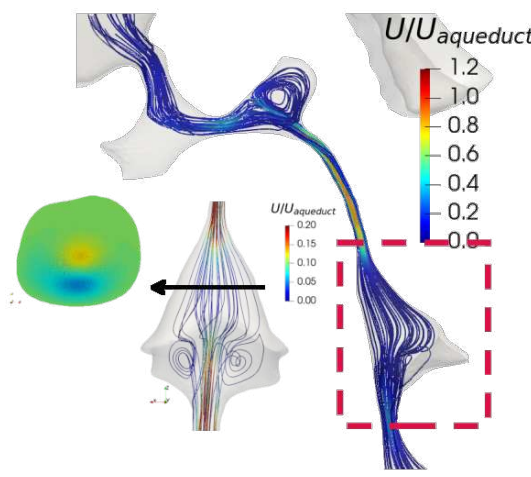

(b) Diastole

Figure 7: Streamlines in the 4th ventricle and contours of the $v$-velocity component at a cross section, the color indicates a velocity magnitude normalized by the mean velocity of the aqueduct of Sylvius, (a) systole phase, (b) diastole phase.

\section{by geometry effects. The flow oscillation in ventricle channels trigger the turbulence transition in the} ventricles. Figure 6 shows the streamlines inside the brain ventricles at a systolic and a diastolic phase. The enlarged images are chosen to focus on the flow motion between the lateral and the 3rd ventricles.

Figure 7 shows the streamlines inside the 4th ventricle at a systolic and a diastolic phase. The cross-

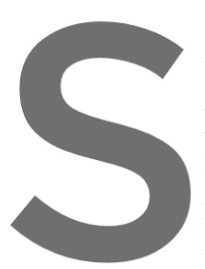
section is located at an axis

the $v$-velocity component, phase two main circulatio induced by the flow passa in the diastolic phase in Fif 7
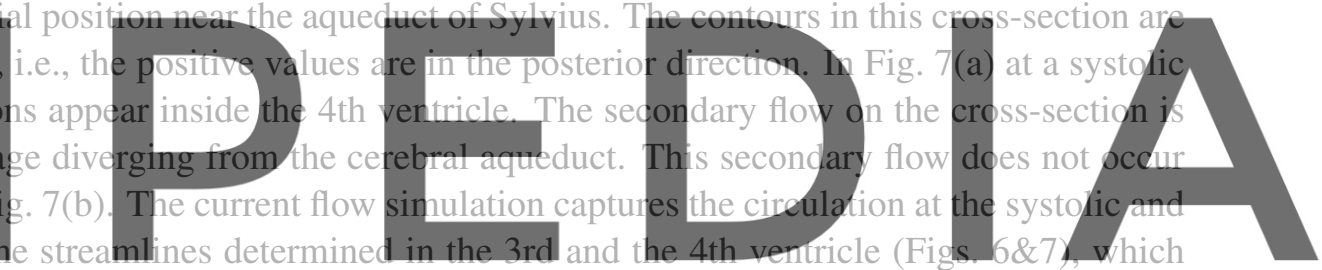

was observed in the measurements using magnetic resonance (MR) velocity mapping ${ }^{17}$.

Register for free at https//www.scipedia.com to download the version without the watermark 6 CONCLUSIONS

- The simulation of a flow oscinlation in brain ventricles was performed by using the lattice Boltzmann (LB) method in the quasi-incompressible regimes for the cerebrospinal fluid motion. The LB method is a powerful numerical solver to complex flow geometry and easily extended to massive parallelization on HPC platforms.

- The geometry of a brain ventricular system was obtained by segmentation of the cerebrospinal fluid which is visualized by the T1-weighted MRI scans. The surface extraction required manual operations to detail the members of central verve system. The main issue was the resolution of the medical images which restricted by the current hardware constraints and practical difficulties. A new approach such as the super resolution via deep learning is necessary to define characteristics and to preserve the contents details in future.

- An oscillating flow boundary condition is defined by the pressure variation at a Womersley number. The CSF motion is captured at the systole and the diastole phase in a cardiac cycle such that the streamline shows a good agreement with the observation based on an MR velocity mapping. 


\section{ACKNOWLEDGEMENTS}

The authors gratefully acknowledge the computing time granted through the Jülich Aachen Research Alliance (JARA) on the supercomputer JURECA ${ }^{29}$ at Forschungszentrum Jülich.

\section{REFERENCES}

[1] Linninger, A., Tangen, K., Hsu, C.-Y., Frim, D. Cerebrospinal fluid mechanics and its coupling to cerebrovascular dynamics. Ann. Rev. Fluid Mech. (2016) 48:219-257.

[2] Dunbar, H. S., Guthrie, T. C., Karpell, B. A. A study of the cerebrospinal fluid pulse wave. Arch. Neurol. (1966) 14(6):624-630.

[3] Hamer, J., Alberti, E., Hoyer, S. et al. Influence of systemic and cerebral vascular factors on the cerebrospinal fluid pulse waves. J. Neurosurg. (1977) 46(1):36-45.

[4] Balédent, O., Gondry-Jouet, C., Meyer, M.-E., De Marco, G., Le Gars, D., et al. Relationship between cerebrospinal fluid and blood dynamics in healthy volunteers and patients with communicating hydrocephalus. Invest. Radiol. (2004) 39(1):45-55.

[5] Armonda, R. A., Citrin, C. M., Foley, K. T., Ellenbogen, R. G. Quantitative cine-mode magnetic resonance imaging of Chiari I malformations: an analysis of cerebrospinal fluid dynamics. Neurosurgery (1994) 35(2):214-224.

[6] Monro, A. Observations on the Structure and Functions of the Nervous System. Edinburgh: William Creech \& Johnson (1783).

[7] Kellie, G. An account of the appearances observed in the dissection of two of three individuals presumed to have perished in the storm of the $3 \mathrm{rd}$, and whose bodies were discovered in the vicinity of Leith on the morning of the 4th, November 1821; with some reflections on the pathology of the brain. Trans. Med. Chir. Soc. Edinburgh (1824) 1:84-169.

[8] Guillaume, J. and Janny, P. Continuous intracranial manometry; importance of the method and first results. Rev. Neurol. (1951) 84:131-142.

[9] Hunter, G. and Smith, H. V. Calcium and magnesium in human cerebrospinal fluid. Nature (1960) 186:161-162.

[10] Hendry, E. B. The osmotic pressure and chemical composition of human body fluids. Clin. Chem. (1962) 8:246-265.

[11] Marmarou, A., Barzo, P., Fatouros, P., et al. Traumatic brain swelling in head injured patients: brain edema or vascular engorgement? Acta. Neurochir. Suppl. Wien (1997) 70:68-70.

[12] Beaumont, A. Physiology of the cerebrospinal fluid and intracranial pressure. Youmans Neurological Surgery, 6th Edition, (2011).

[13] Czosnyka, M., Czosnyka, Z., Balédent, O., Weerakkody, R., Kasprowicz, M., Smielewski, P., Pickard, J. Dynamics of cerebrospinal fluid: from theoretical models to clinical applications. Biomechanical Engineering of the Brain, Editor: Miller, K., Springer (2011).

[14] Gupta, S., Soellinger, M., Grzybowski, D. M., Boesiger, P., Biddiscombe, J., Poulikakos, D., Kurtcuoglu, V. Cerebrospinal fluid dynamics in the human cranial subarachnoid space: an 
overlooked mediator of cerebral disease. I. computational model. J. Roy. Soc. Interface (2010) 7(49):1195-1204.

[15] Tangen, K., Hsu, Y., Zhu, D., Linninger, A. CNS wide simulation of flow resistance and drug transport due to spinal microanatomy. J. Biomech. (2015) 48(10):2144-2154.

[16] Khani, M., Sass, L. R., Xing, T., Sharp, M. K., Balédent, O., Martin, B. A. Anthropomorphic model of intrathecal cerebrospinal fluid dynamics within the spinal subarachnoid space: spinal cord nerve roots increase steady-streaming. J. Biomech. Eng. (2018) 140(8):081012-1.

[17] Stadlbauer, A., Salomonowitz, E., van der Riet, W., Buchfelder, M., Ganslandt, O. Insight into the patterns of cerebrospinal fluid flow in the human ventricular system using MR velocity mapping. NueroImage (2010) 51(1):42-52.

[18] Freitas, R., Henze, A., Meinke, M., Schröder, W. Analysis of Lattice-Boltzmann methods for internal flows. Comput. Fluids (2011) 47(1):115-121.

[19] Eitel-Amor, G., Meinke, M., Schröder, W. A lattice-Boltzmann method with hierarchically refined meshes. Comput. Fluids (2013) 75:127-139.

[20] Lintermann, A., Meinke, M., Schröder, W. Fluid mechanics based classification of the respiratory efficiency of several nasal cavities. Comput. Biol. Med. (2013) 43(11):1833-1852.

[21] Lintermann, A., Schröder, W. A hierarchical numerical journey through the nasal cavity: from nose-like models to real anatomies. Flow Turbul. Combust. (2019) 102(1):89-116.

[22] Bhatnagar, P. L., Gross, E. P., Krook, M. A model for collision processes in gases. i. small amplitude processes in charged and neutral one-component systems. Phys. Rev. (1954) 94(3):511-525.

[23] Qian, Y. H., D’Humières, D., Lallemand, P. Lattice BGK Models for Navier-Stokes equation. Europhysics Letters (EPL) (1992) 17(6):479-484.

[24] Benzi, R., Succi, S., Vergassola, M. The lattice Boltzmann equation: theory and applications. Phys. Rep. (1992) 222(3):145-197.

[25] Lintermann, A., Schlimpert, S., Grimmen, J. H., Günther, C., Meinke, M., Schröder, W. Massively parallel grid generation on HPC systems. Comput. Method Appl. M. (2014) 277:131-153.

[26] Fedorov A., Beichel R., Kalpathy-Cramer J., Finet J., Fillion-Robin J-C., Pujol S., Bauer C., Jennings D., Fennessy F., Sonka M., Buatti J., Aylward S.R., Miller J.V., Pieper S., Kikinis R. 3D Slicer as an image computing platform for the quantitative imaging network. Magnetic Resonance Imaging (2012) 30(9):1323-41. PMID: 22770690.

[27] Ahmed, S. A., Giddens, D. P. Pulsatile poststenotic flow studies with Laser Doppler Anemometry. J. Biomech. (1984) 17(9):695-705

[28] Gholampour, S. FSI simulation of CSF hydrodynamic changes in a large population of noncommunicating hydrocephalus patients during treatment process with regard to their clinical symptoms. PLoS ONE (2018) 13(4): 0196216.

[29] Krause, D. and Thörnig, P. JURECA: Modular supercomputer at Jülich Supercomputing Centre. J. Large-Scale Research Facilities (2018) 4:A132. 